\title{
Preface to the special issue on "Practical methods and rigorous theories in numerical algebra and scientific computing"
}

\author{
Zhong-Zhi Bai · Iain S. Duff
}

Received: 22 December 2014 / Accepted: 22 December 2014 / Published online: 12 March 2015

(C) Springer Science+Business Media Dordrecht 2015

The Fourth International Conference on Numerical Algebra and Scientific Computing (NASC 2012) was held in Dalian from 20 to 24 October, 2012. More than 160 participants attended the conference, coming from many countries including Canada, China, Czech Republic, France, Italy, Japan, Russia, Serbia, Sweden, the UK, and the USA. This issue of the Journal of Engineering Mathematics contains carefully selected and regularly refereed papers by participants of the conference.

The conference was organized by the Chinese Academy of Sciences supported by Dalian University of Technology who were in charge of the local organization. It was chaired by Lev A. Krukier, the Southern Federal University, Russia, and by Zhong-Ci Shi, the Chinese Academy of Sciences, China. The organizing committee consisted of Zhong-Zhi Bai from the Chinese Academy of Sciences, Beijing; Xiu-Ping Liu, Zhong-Xuan Luo, Zhi-Xun Su, Wei $\mathrm{Wu}$ and Bo Yu, all from Dalian University of Technology, Dalian; and Zhi-Ru Ren also from the Chinese Academy of Sciences, Beijing.

The NASC series of conferences involve top level researchers in numerical algebra and scientific computing from all over the world. In addition to the formal presentations on state-of-the art research, these conferences enable the participants to exchange new ideas and discuss future developments as well as providing a platform for communication and networking. The talks and the refereed subset of invited and contributed submissions in this volume demonstrate the diversity of work in this area. The topics discussed at NASC 2012 include direct and iterative methods for systems of linear and nonlinear equations; preconditioning and iterative methods for least-squares problems; matrix multisplitting iteration methods for linear complementarity problems; inner-outer and two-stage strategies; alternating matrix splitting iterations; extrapolation techniques; conditioning and spectral properties of matrices; Tikhonov regularization and modifications; sparse and structured matrix computations in saddle-point problems, model updating problems, state feedback pole assignment problems, and quadratic and inverse eigenvalue problems; effective and robust solvers for image restoration, network analysis, multiphase flow simulations, and the three-dimensional photonic crystals, as well as parallel computing. Applications to ordinary

\section{Z.-Z. Bai (凶)}

State Key Laboratory of Scientific/Engineering Computing, Institute of Computational Mathematics and Scientific/Engineering Computing, Academy of Mathematics and Systems Science, Chinese Academy of Sciences, P.O. Box 2719, Beijing 100190, People's Republic of China

e-mail: bzz@1sec.cc.ac.cn

I. S. Duff

R18, Rutherford Appleton Laboratory, Harwell Oxford Campus, Didcot, Oxon OX11 0QX, England, UK e-mail: iain.duff@stfc.ac.uk 
and partial differential equations, time-delay systems, dimensionality reduction and feature extraction, automatic differentiation, American pricing options and constrained global optimization, were also presented.

NASC is a series of international conferences organized by the Academy of Mathematics and Systems Science (AMSS), the Chinese Academy of Sciences, and run by the Chinese Numerical Algebra Group (CNAG), with the first meeting in 2006. This conference series highlights recent advances in theoretical, computational and practical aspects of linear and nonlinear numerical algebra. The first meeting (NASC 2006) was held in AMSS at Beijing, October 22-25, 2006; the second (NASC 2008) was held in Nanjing Normal University, November 2-5, 2008; and the third (NASC 2010) was held at AMSS in Beijing, October 23-27, 2010. The special issues of these three conferences were published in the Journal of Computational and Applied Mathematics 226:1(2009), Linear Algebra and Its Applications 434:11(2001) and Numerical Linear Algebra with Applications 19:6(2012), respectively.

Finally, we would like to thank Professor Stephen K. Wilson, the joint Editor-in-Chief of the journal, for agreeing to and helping with the publication of this special issue. We would also like to take this opportunity to thank the sponsors of the conference: the Academy of Mathematics and Systems Science, Chinese Academy of Sciences; Dalian University of Technology; and the National Natural Science Foundation of China. Our special thanks go to Prof. Bo Yu, Dr. Zhi-Ru Ren, and Ms. Min Wei (AMSS, Chinese Academy of Sciences) and others, for their enthusiasm and help with the organization of the conference. 